\title{
Application of the phase method in radioisotope measurements of the liquid - solid particles flow in the vertical pipeline
}

\author{
Robert Hanus $^{1, \mathrm{a}}$, Marcin Zych ${ }^{2}$, Leszek Petryka $^{2}$, Volodymyr Mosorov ${ }^{3}$ and Paweł Hanus ${ }^{2}$ \\ ${ }^{1}$ Rzeszow University of Technology, Rzeszow 35-959, Poland \\ ${ }^{2}$ AGH University of Science and Technology, Krakow 30-059, Poland \\ ${ }^{3}$ Lodz University of Technology, Lodz 90-924, Poland
}

\begin{abstract}
The paper presents idea and an application of the gamma-absorption method to a two-phase flow investigation in a vertical pipeline, where the flow of solid particles transported by water was examined by a set of two ${ }^{241} \mathrm{Am}$ radioactive sources and probes with $\mathrm{NaI}(\mathrm{Tl})$ scintillation crystals. In the described experiments as solid phase the ceramic models representing natural polymetallic ocean nodules were used. For advanced analysis of electrical signals obtained from detectors the phase of cross-spectral density function has been applied. Results of the average solid-phase velocity measurements were compared with one obtained by application of the classical cross-correlation. It was found that the combined uncertainties of the velocity of solid particles evaluation in the presented experiment did not exceed $0.6 \%$ in phase method and $3.2 \%$ in crosscorrelation method.
\end{abstract}

\section{Introduction}

Two-phase liquid-solid particles flow often occur in the mining industry. An example would be vertical pipeline hydrotransport of minerals, e.f. polymetallic nodules. Nodules are porous organic and mineral compositions which contain a various metals (mostly $\mathrm{Mn}, \mathrm{Si}, \mathrm{Fe}, \mathrm{Al}$, $\mathrm{Na}, \mathrm{Mg}, \mathrm{Ni}, \mathrm{K}, \mathrm{Cu}$ ) [1 - 4]. They occur on the bottom of seas and oceans, but the richest deposits can be found at the Pacific Ocean bed below of about $5.000 \mathrm{~m}$ depth in the so called Clarion-Clipperton zone [ibid]. The ocean nodules usually have the form of irregular grains with the diameter of several up to $0.5 \mathrm{~m}$ and density of about $2 \mathrm{~g} / \mathrm{cm}^{3}$ in wet state. The mining of nodules using the hydraulic method requires grain vertical transport by water to sea level in extremely hard and varying environment. Determination of the velocity of nodules of various sizes in the vibrating pipeline at a significant depth is a very difficult task and requires the use noninvasive measurement techniques.

One of these techniques, which are employed for many years in measurements of two-phase flows in pipelines and open channels, is a method using radioactive isotopes. In this method radiotracer is injected under certain conditions into the flow or sealed radioactive sources are used [5-13]. In both cases as detectors of radiation the scintillation probes are mounted outside the analyzed stream. The advantage of such methods is the simplicity and relatively high accuracy.
Typically in such measurements the mutually delayed stochastic signals are provided by scintillation probes situated on the walls of the pipeline. Measured time delay of signals is applied to determine the averaged velocity of the minority phase of the two-phase flow and other parameters. The processing of these signals requires the use of it conditioning and statistical methods in the time or frequency domain. The cross-correlation function (CCF) is the most known methods of time delay estimation applied for stationary random signals [12 16]. Other methods in time domain includes differential methods [17], or one based on the conditional averaging of signals [18 - 20], as well as combination of the above and CCF $[21,22]$ or cross-correlation analysis with the Hilbert Transform [14, 23, 24]. In the frequency domain the phase of cross-spectral density function (CSDF) or the spectral density of differential signal can be used [14, 25 - 28]. The paper presents an application of the phase method to radioisotope measurement of the mean velocity of solid particles transported by water in a vertical pipeline.

The paper consists of five sections. In the second one an idea of the application of gamma-absorption for liquid-solid particles flow evaluation and the laboratory installation is briefly described. The section 3 presents basic principles of time delay estimation applying phase of cross-spectral density function. In section 4 an exemplary results of time delay and average velocity measurements using phase method in comparison of CCF

\footnotetext{
$\bar{a}$ Corresponding author: rohan@prz.edu.pl
} 
are presented. The section 5 contains the final conclusions.

\section{Application of gamma-ray absorption to liquid-solids flow investigation}

The basic principle of gamma-ray absorption measurement is based on fact that the intensity of a collimated gamma beam decreases as it passes through matter [8].

The principle and typical gamma-absorption measuring equipment for liquid-solid particles flow evaluation in a vertical pipeline is presented in figure 1 .

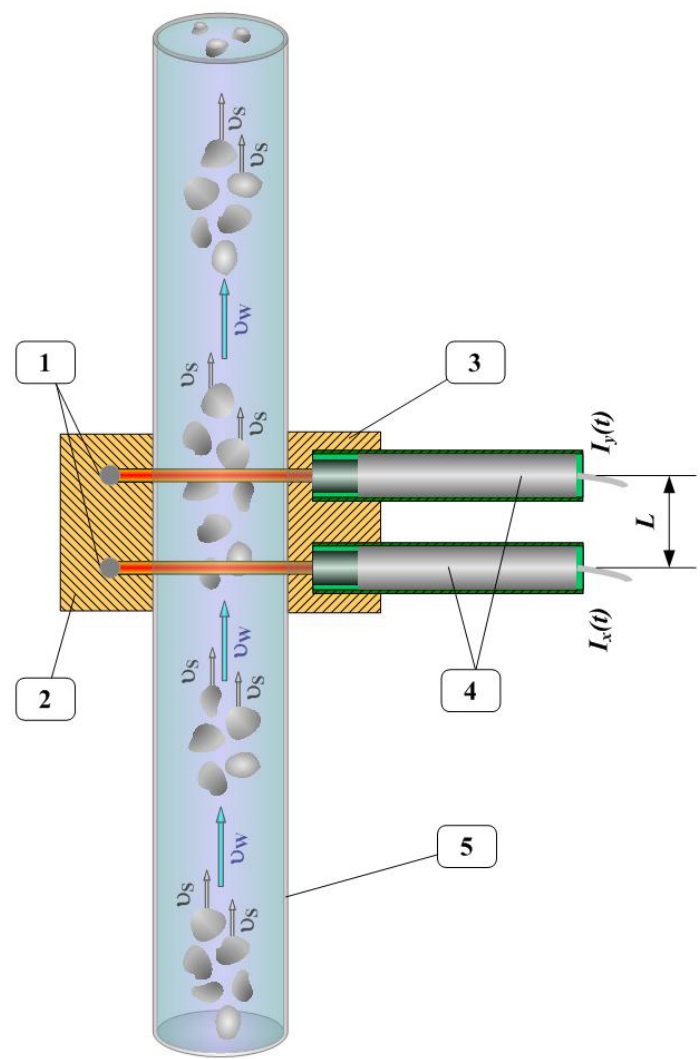

Figure 1. The gamma-ray absorption measuring equipment: 1 - gamma radiation source, 2 - collimator of source, 3 - collimator of detector, 4 - scintillation probe, 5 - pipeline, $v_{S}, v_{W}-$ velocities of solid particles and water respectively.

Two sealed radioactive sources (1) emit $\gamma$ radiation beams shaped by the collimator (2). Photons pass through the pipeline (5) with analyzed mixture. The changes of the intensity of the radiation are recorded by the scintillation probes (4) with collimators (3) and next converted into output electrical impulses [7]. Count rates $I_{x}$ and $I_{y}$ at the outputs of probes depend on condition of the flowing medium in the cross section.

The measuring set presented in figure 1 was applied in the experimental laboratory stand built up in the Water Laboratory of the Wroclaw University of Environmental and Life Sciences for investigation of the polymetallic nodules hydrotransport. The basic part of the installation is a vertical pipeline of the length of $7.75 \mathrm{~m}$ with an internal diameter of $150 \mathrm{~mm}$ made of acrylic glass. The laboratory stand was in detail described in papers $[11,13$, 21]. The view of some part of the experimental installation with measuring section of the pipe and mounted gamma-absorption set is shown in figure 2 .

In the investigations a linear ${ }^{241} \mathrm{Am} \gamma$-ray sources with an activity of $100 \mathrm{mCi}$ and probes with 2 " $\mathrm{NaI}(\mathrm{Tl})$ scintillation crystal are used. Two such sets were located on the pipe with the distance of $L=90 \mathrm{~mm}$ between them.

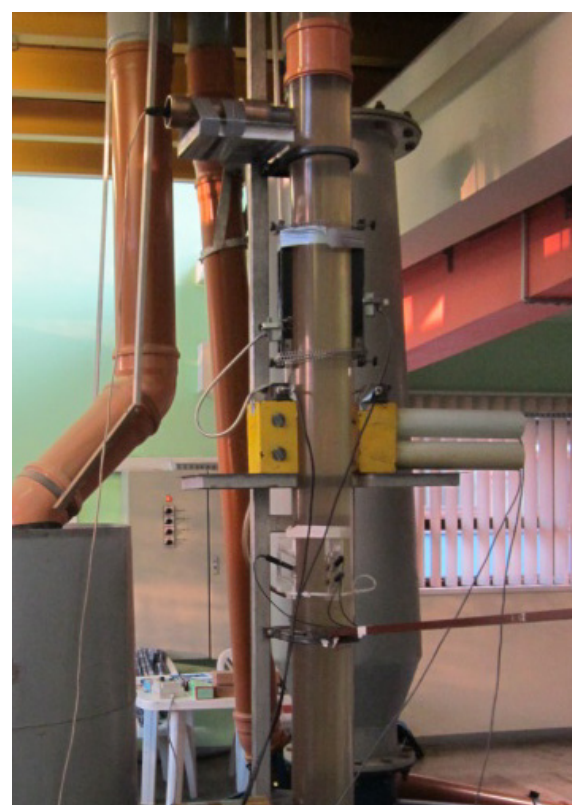

Figure 2. View of some part of the laboratory stand built up in the Water Laboratory of the Wroclaw University of Environmental and Life Sciences (Poland).

The geometry of applied gamma-ray absorption measurement is shown in figure 3 , where all dimensions are given in $\mathrm{mm}$.

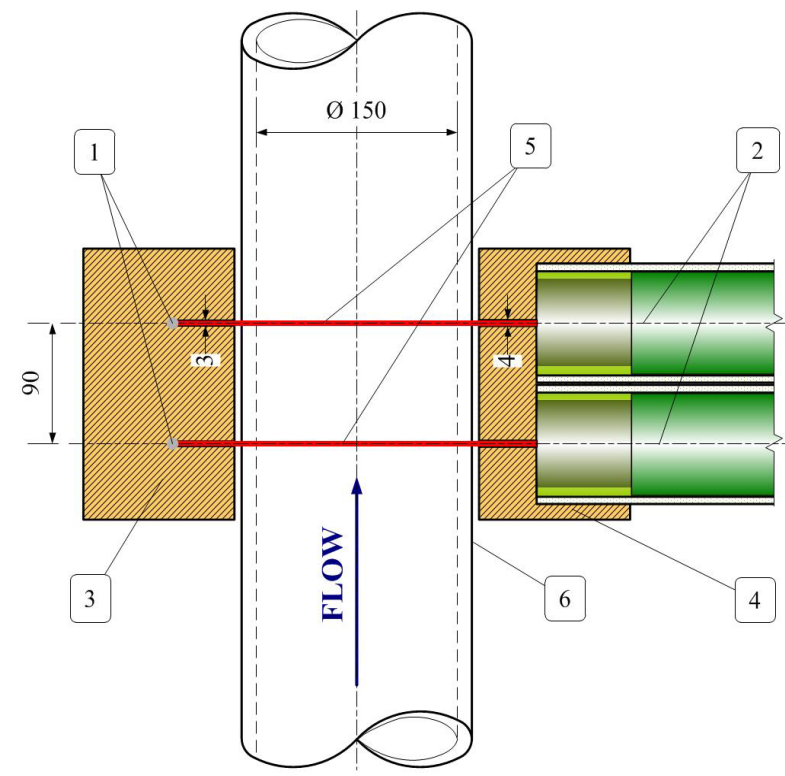

Figure 3. The geometry of $\gamma$-ray absorption measurement, where all dimensions are given in mm.: $1-{ }^{241} \mathrm{Am}$ source, 2 - scintillation probe with 2 " $\mathrm{NaI}(\mathrm{Tl})$ crystal, 3 - collimator of sources, 4 - collimator of detectors, 5 - gamma-ray beam, 6 -pipeline. 
The data acquisition equipment is comprised of the dedicated 8-channel EC Electronics counter of HSC 8000 connected to PC using an USB port. Voltage pulses $I_{x}$ and $I_{y}$ counted within the selected sampling time $\Delta t=1 \mathrm{~ms}$ create mutually delayed discrete stochastic signals. An exemplary time records of signals (after centering and removal a background contribution) obtained in the WRQ051 experiment are presented in figure 4.
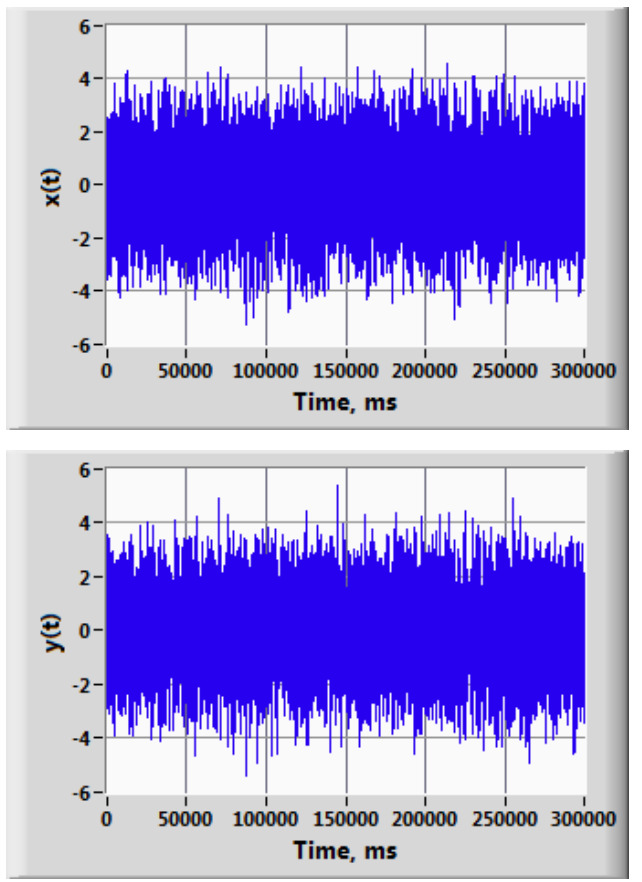

Figure 4. Time records of signal $x(t)$ and $y(t)$ (after preprocessing) obtained in the WRQ051 experiment.

\section{The analysis of signals}

The signals recorded at edges of $L$ allow determination of the $\tau_{0}$ time delay (transit time) necessary for transportation of the solid phase through the measuring section of the pipe. Then the average velocity of solid particles flow $v_{S}$ is calculated from the formula:

$$
v_{S}=L / \tau_{0} .
$$

The frequently used method of time delay estimation of the ergodic random signals $x(t)$ and $y(t)$ is based on the cross - correlation function $R_{x y}(\tau)$, defined by equation:

$$
R_{x y}(\tau)=\lim _{T \rightarrow \infty} \frac{1}{T} \int_{0}^{T} x(t) \cdot y(t+\tau) d t
$$

where $T$ is the averaging time, $\tau$ - time delay $[14,16]$.

The $\tau_{0}$ transportation time delay is determined on base of position of the CCF main maximum.

To time delay estimation in the frequency domain the phase of cross-spectral density function (CSDF) can be applied. Determined in practice one-sided CSDF for the frequency range $0<f<\infty$ is expressed by the following formula [14]:

$$
\underline{G}_{x y}(f)=2 \int_{-\infty}^{\infty} R_{x y}(\tau) \cdot e^{-j 2 \pi f \tau} d \tau
$$

Normalized CSDF is referred to as $\gamma_{x y}(f)$ coherence function. In practice, the real-valued squared coherence is used:

$$
\gamma_{x y}^{2}(f)=\frac{\left|\underline{G}_{x y}(f)\right|^{2}}{G_{x x}(f) \cdot G_{y y}(f)},
$$

where $G_{x x}(f)$ and $G_{y y}(f)$ are autospectral density functions of signals $x(t)$ and $y(t)$ respectively.

Consequently the squared coherence $\gamma_{x y}^{2}(f)$ satisfies the condition:

$$
0 \leq \gamma_{x y}^{2}(f) \leq 1 .
$$

The $\Phi_{x y}(f)$ phase of the CSDF is a linear function of frequency $f$ :

$$
\Phi_{x y}(f)=\operatorname{arctg}\left\{\frac{\operatorname{Im}\left[\underline{G}_{x y}(f)\right]}{\operatorname{Re}\left[\underline{G}_{x y}(f)\right]}\right\}=2 \pi \cdot f \cdot \tau_{0} .
$$

For calculation of spectral densities the discrete Fourier transform (DFT) or it fast version FFT is applied directly for the sequences of the discrete signal $x(n)$ and $y(n)$, where $n=t / \Delta t$. In spectral analysis of stochastic signals an averaging of the obtained spectral density is necessary. Often used Welch procedure requires the sets of signal samples to be divided for $N_{d}$ windowing segments, each of the $N$ samples. The $N_{d}$ value should be big enough to limit the statistical fluctuations of CSDF estimation [14]. The use of the discrete Fourier transform gives the $k$ useful frequencies $f_{k}(k=0 \ldots(N-1) / 2)$, and their spectrum resolution is $\Delta f=1 / N \Delta t$. Consequently the transport delay is determined from the smoothed $\widetilde{\Phi}_{x y}(f)$ estimator after applying the procedure of the phase unwrapping. Figure 5 presents the graph of the $\widetilde{\Phi}_{x y}(f)=f(f)$ function determined in the WRQ051 experiment $\left(N_{d}=360, N=500\right.$, and Hamming window).

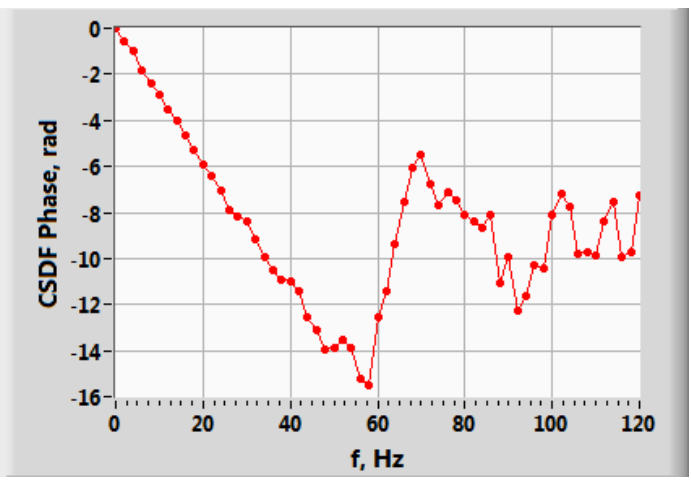

Figure 5. Plot of the smoothed CSDF phase. 
Because function $\widetilde{\Phi}_{x y}(f)$ is nominally linear and passes through the coordinate origin, the $\hat{\tau}_{0}$ time delay estimator can be found out by the $a$ slope of the regression line fitted to the CSDF phase against frequency:

$$
\hat{\tau}_{0}=-\frac{1}{2 \pi} \cdot \hat{a}=-\frac{1}{2 \pi}\left[\sum_{k=1}^{m} f_{k} \cdot \widetilde{\Phi}_{x y}\left(f_{k}\right) / \sum_{k=1}^{m} f_{k}^{2}\right],
$$

where $m$ is the number of the pairs of both phase and frequency values used for the regression analysis [25]. The selection of the appropriate frequency linearization range is vital due to fluctuations of the phase characteristics above the cut-off frequency band of the recorded signals. In this case the cut-off frequency can be determined on the significant value of coherence function. Figure 6 presents the graph of the smoothed $\widetilde{\gamma}_{x y}^{2}(f)$ function obtained in the WRQ051 experiment.

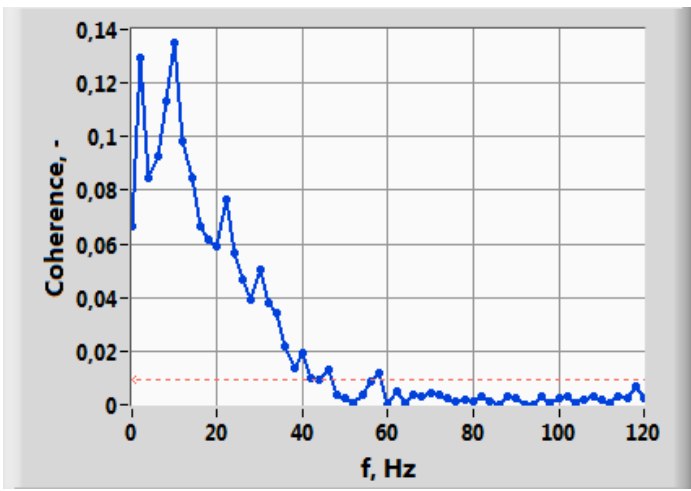

Figure 6. Smoothed coherence function.

Dotted line in the figure 6 marked the border of one-sided confidence interval $E_{\alpha}$ fixed for the significance level $\alpha=0.05$ from the relation [29]:

$$
E_{\alpha}=(1-\alpha)^{1 /\left(N_{d}-1\right)}
$$

Based on the $E_{\alpha}$ value, the cut-off frequency and $m$ for $\hat{\tau}_{0}$ time delay estimation are determined.

\section{Exemplary results}

The graph of the CSDF phase obtained in the WRQ051 experiment for the selected frequency range is shown in figure 7 . The solid line represents the fitted regression function determined to find out the transportation time delay estimator from (7).

The standard uncertainty $u\left(\hat{\tau}_{0}\right)$ calculated using phase CSDF is given by [27]:

$$
u\left(\hat{\tau}_{0}\right)=\left\{\frac{1}{4 \pi^{2} \cdot(m-1) \cdot \sum_{k=1}^{m} f_{k}^{2}}\left[\sum_{k=1}^{m} \widetilde{\Phi}_{x y k}^{2}\left(f_{k}\right)-\frac{\left[\sum_{k=1}^{m} f_{k} \cdot \widetilde{\Phi}_{x y k}\left(f_{k}\right)\right]^{2}}{\sum_{k=1}^{m} f_{k}^{2}}\right]\right\}^{\frac{1}{2}}
$$

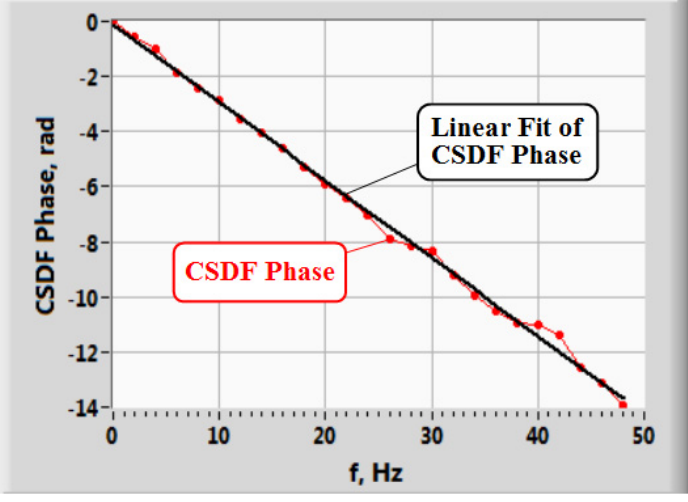

Figure 7. Linear fit of the smoothed CSDF phase determined in the WRQ051 experiment.

In table 1 the results of the time delay estimation using phase method and its standard uncertainty obtained in the WRQ051 experiment for $m=48$ are presented. For comparison, the results obtained under analysis of the CCF distribution are also given [21].

Table 1. Results of the time delay estimation in the WRQ051 experiment.

\begin{tabular}{|c|c|c|}
\hline Method & $\begin{array}{c}\text { Time delay } \\
\hat{\tau}_{0}, \mathrm{~ms}\end{array}$ & $\begin{array}{c}\text { Standard } \\
\text { uncertainty } \\
u\left(\hat{\tau}_{0}\right), \mathrm{ms}\end{array}$ \\
\hline CSDF phase & 45.23 & 0.26 \\
\hline $\mathrm{CCF}$ & 45.42 & 1.44 \\
\hline
\end{tabular}

The combined standard uncertainty $u_{c}\left(v_{S}\right)$ of the solid particles average velocity, with negligible small uncertainties of the acquisition set, depends on an inaccuracy of uncorrelated $L$ and $\hat{\tau}_{0}$ determination:

$$
u_{c}\left(v_{S}\right)=\left[\left(\frac{\partial v_{S}}{\partial L}\right)^{2} \cdot u^{2}(L)+\left(\frac{\partial v_{S}}{\partial \hat{\tau}_{0}}\right)^{2} \cdot u^{2}\left(\hat{\tau}_{0}\right)\right]^{1 / 2},
$$

where $u(L)$ is the standard uncertainty of the distance between detectors.

Results of the average velocity $v_{S}$ and its combined uncertainty $u_{c}\left(v_{S}\right)$ [30] obtained in the WRQ051 experiment by use phase and CCF methods are presented in the table 2.

Table 2. Average velocity of air $v_{S}$ and its uncertainty $u_{c}\left(v_{S}\right)$.

\begin{tabular}{|c|c|c|}
\hline Method & $\begin{array}{c}\text { Average velocity } \\
v_{S}, \mathrm{~m} / \mathrm{s}\end{array}$ & $\begin{array}{c}\text { Combined } \\
\text { uncertainty } \\
u_{c}\left(v_{S}\right), \mathrm{m} / \mathrm{s}\end{array}$ \\
\hline CSDF phase & 1.990 & 0.011 \\
\hline $\mathrm{CCF}$ & 1.982 & 0.063 \\
\hline
\end{tabular}




\section{Conclusions}

Based on the research carried out and obtained results, it can be stated that the CSDF phase method may be successfully applied to such an investigation as nodules vertical pipeline hydrotransport employing the radioisotope techniques. It was found that the combined uncertainties of the average velocity of solid particles in the exemplary the WRQ051 experiment did not exceed $0.6 \%$ (phase CSDF) and 3.2\% (CCF) levels. Moreover, in the cross-correlation method, the filtration of the recorded signals may be additionally used in order to reduce noises [11]. On contrary to CCF metod, the filtration of signals in the phase method is not necessary.

\section{Acknowledgment}

The authors would like to thank teams of prof. Jerzy Sobota and prof. Pavel Vlasak for their cooperation during the measurements undertaken at Wroclaw University of Environmental and Life Sciences.

\section{References}

1. D.S. Cronan (ed.), Handbook of marine mineral deposits (CRC Press, Boca Raton, 2000)

2. R. Kotliński, http://www.iom.gov.pl/uploads/iom attachment-95635.pdf

3. S. Depowski, et al, Mineral resources of the seas and oceans (SCHOLAR, Warsaw, 1998)

4. http://www.iom.gov.pl/

5. G. Falcone, G.F. Hewitt, C. Alimonti, Multiphase flow metering: principles and applications (Elsevier, Amsterdam, 2009)

6. G.A. Johansen, P. Jackson, Radioisotope gauges for industrial process measurements (Wiley, New York, 2004)

7. Z. Yu, B. Qincheng, H. Richa, Appl. Therm. Eng. 60, 398-410 (2013)

8. W.A.S. Kumara, B.M. Halvorsen, M.C. Melaaen, Int. J. Multiphas. Flow 36, 467-480 (2010)

9. B.K. Arvoh, R. Hoffmann, M. Halstensen, Flow
Meas. Instrum. 23, 56-65 (2012)

10. M. Zych, et al., Flow Meas. Instrum. 35, 11-15 (2014)

11. L. Petryka, R. Hanus, M. Zych, Prz. Elektrotech. 88 (1b), 185-188 (2012)

12. S.H. Jung, et al, Appl. Rad. Isotop. 67, 1254-1258 (2009)

13. L. Petryka, et al, Proc. 10th ISOPE Ocean Mining \& Gas Hydrates Symp. OMS-2013, 230-233 (2013)

14. J.S. Bendat, A.G. Piersol, Random data - analysis and measurement procedures. (Wiley, New York, 2010)

15. S.L. Soo (ed.), Instrumentation for fluid-particle flow. (Noyes Publications, New Jersey, 1999)

16. M.S. Beck, A. Plaskowski, Cross correlation flowmeters - their design and application. (Adam Hilger, Bristol, 1987)

17. G. Jacovitti, G. Scarano, IEEE Trans. Sign. Proc 41, 525-533 (1993)

18. R. Hanus, Prz. Elektrotech. 86 (6), 232-235 (2010)

19. A. Kowalczyk, R. Hanus, A. Szlachta, Metrol. Meas. Syst. 18, 335-342 (2011)

20. R. Hanus, et al, Proc. 2012 IEEE Mediterranean Electrotechnical Conference MELECON 2012, 144 147 (2012)

21. R. Hanus, L. Petryka, M. Zych, Flow Meas. Instrum. 40, 58-63 (2014)

22. R. Hanus, M. Zych, L. Petryka, JPCS 530012042 (2014)

23. R.C. Cabot, IEEE Trans. Acoust. Speech Sign. Proc. 29, 607-609 (1981)

24. R. Hanus, Prz. Elektrotech. 88 (10b), 39-41 (2012)

25. A.G. Piersol, IEEE Trans. Acoust. Speech Sign Proc. 29, 471-477 (1981)

26. V. Mosorov, Electr. Lett. 42, 630-632 (2006)

27. R. Hanus, L. Petryka, M. Zych, MAaM 58 (3), 236239 (2012)

28. R. Hanus, Prz. Elektrotech. 84 (12), 301-303 (2008)

29. D.M. Halliday, et al, Prog. Biophys. Mol. Bio. 64, 237-278 (1995)

30. Guide to the expression of uncertainly in measurement (International Organisation for Standardisation, 1995) 